\title{
Anti-inflammatory effects of gambogic acid in murine collagen-induced arthritis through PI3K/Akt signaling pathway
}

\author{
QING LAI WANG, DONG ZHEN YANG and CUNXIAN LV \\ Department of Traditional Chinese Medicine Orthopaedics, Chinese Traditional \\ Medicine Hospital of Wenzhou, Wenzhou, Zhejiang 325000, P.R. China
}

Received March 29, 2017; Accepted September 5, 2017

DOI: $10.3892 / \mathrm{mmr} .2018 .8389$

\begin{abstract}
Garcinia angustifolia is a dry resin secreted by Garcinia cambogia, which has the functions of breaking blood, detoxifying, stopping bleeding and killing insects. It is used for the treatment of cancer and brain edema. Gambogic acid is the primary active ingredient. The present study aimed to investigate the anti-inflammatory and antiproliferative effects of gambogic acid on arthritis and the possible mechanisms. It was demonstrated that gambogic acid decreased arthritic scores in murine collagen-induced arthritic mice. The tumor necrosis factor (TNF)- $\alpha$, interleukin (IL)-1 $\beta$, IL-6 and IL-18 concentrations, and caspase- 3 and caspase- 9 were significantly inhibited by gambogic acid in arthritic mice. Gambogic acid decreased matrix metalloproteinases (MMP)-2, MMP-9, nuclear factor (NF)- $\kappa \mathrm{B}$ and phosphorylated-p38 protein expression, and increased tissue inhibitors of matrix metalloproteases-1 (TIMP-1) protein expression in arthritic mice. Furthermore, the phosphoinositide 3-kinase (PI3K)/AKT serine/threonine kinase (Akt) signaling pathway was induced in arthritic mice treated with gambogic acid. The results suggested that gambogic acid induced anti-inflammatory effects in murine collagen-induced arthritis, through the PI3K/Akt signaling pathway, and offers future potential for application in arthritis patients.
\end{abstract}

\section{Introduction}

Rheumatoid arthritis (RA) is a systemic autoimmune disease characterized by polyarticularsynovitis (1). Its autoantigen is not organ-specific, but a common component of many organs and tissues, such as nucleus, mitochondria and so on, so it can cause the damage of multiple organs and multiple tissues (2).

Correspondence to: Mr Cunxian Lv, Department of Traditional Chinese Medicine Orthopaedics, Chinese Traditional Medicine Hospital of Wenzhou, 9 Jiaowei Road, Wenzhou, Zhejiang 325000, P.R. China

E-mail: 1wef70004114@126.com

Key words: gambogic acid, arthritis, PI3K/Akt, anti-inflammatory effects
Epidemiological survey shows that in China, the incidence rate of RA is 0.32-0.38\%, while in Europe, the United States and Africa, it is up to $0.5-2.0 \%$. Its pathogenesis is complex, influenced by genetic and environmental factors (3). As the patients with RA have high disability rate, it brings a great financial burden to the patients themselves and the whole community, which has becomethe hot topic during the last 10 years for domestic and foreign scholars (3). The basic pathologic changes of RA are synovial cell hyperplasia, thickening of lining layer, infiltration of multiple inflammatory cells, pannus formation and destruction of cartilage and bone tissue, eventually leading to joint deformity and loss of function (4).

RA is related to the signal transduction pathways of multiple cytokines. In recent years, it has been found that phosphoinositide 3 kinase (PI3K)/protein kinase B (PKB) signaling pathway ( $\mathrm{PI} 3 \mathrm{~K} / \mathrm{Akt}$ pathway) is involved in a very rapid signal transduction system from the membrane to the nuclear and its downstream pathways Bad, caspase and nuclear factor $(\mathrm{NF})-\kappa \mathrm{B}$ are important pathways affecting osteoarthritis (5). Recent studies have shown that the signal transduction pathway ofkinase plays an important role in the process of chondrocyte apoptosis $(5,6)$.

$\mathrm{PI} 3 \mathrm{~K} / \mathrm{Akt} / \mathrm{mTOR}$ signal pathway plays an important role in the proliferation and survivalof lymphocyte (7). However, the literature has shown that PI3K/Akt/mTOR signal transduction pathway isstudied in the pathological mechanisms and treatment methods of malignant tumors. mTOR and PI3K signals play an important role in regulation of B lymphocyte morphology, metabolic activity, and cell cycle progression (8).

Gamboge is adry resinsecreted by Garcinia plant, Garcinia hanbaryi Hook. f. It is produced mainly in Cambodia, India, Thailand and Vietnam, as well as Guangdong and Hainan in China (9). It has been documented for a long time in our traditional medicine, as Garcinia cold, Pickle, Sim, astringent, toxic, with the functions of breaking blood, detoxifying, stopping bleeding and killing insects, which has been used for the treatment of crewels, scrofulaceum, and carbuncle, since ancient times (10). It contains gambogic acid, neogambogic acid, allogambogic acid, morellin, isomorellin, morellic acid, isomorellic acid and so on, in which gambogic acid is the main active ingredient. Gambogic acid (Fig. 1) has anti-inflammatory, antioxidation and anticancer effects $(11,12)$. Ma et al $(13)$ showed that gambogic acid inhibits osteoclast formation and ovariectomy-induced osteoporosis. The purpose of this study is for the first time to 
elucidate the anti-inflammatory and antiproliferative effects of gambogic acid on arthritis and the possible mechanisms.

\section{Materials and methods}

Animals and treatment. Collagen-induced arthritis in mice was a common RA model using collagen II. Male DBA/1 mice (5-week-old) was purchased from Wenzhou Laboratory Animal Center (Wenzhou, China) and kept in specific pathogen-free conditions in Animal Center of Wenzhou Medical University. This study gained ethical approval from Chinese Traditional Medicine (Wenzhou, China). Arthritis was induced and emulsified in complete Freund's adjuvant and injected intradermally into the tail with $200 \mu \mathrm{g}$ of collagen II (14). Meanwhile, in treatment with gambogic acid group, mice were treatment with $2 \mathrm{mg} / \mathrm{kg} / 3$ day (intraperitoneal injection) for 28 days (15). All mice were randomly assigned into three groups: Sham group, RA model group and GA group; amount of very group is 10 mice.

ELISA analyzing. Serum from very mice after treatment with gambogic acid at 28 days was collected and centrifuged at $1,500 \mathrm{~g}$ for $10 \mathrm{~min}$. Serum was used to analyze inflammation factors [tumor necrosis factor (TNF)- $\alpha$, interleukin (IL)- $1 \beta$, IL-6 and IL-18 concentrations] using ELISA kits (Nanjing Jiancheng Biology Engineering Institute, Nanjing, China).

Then, tissue extracts were prepared from very mice after treatment with gambogic acid at 28 days and extracted using RIPA assay. Tissue extracts were prepared after treatment with gambogic acid at 28 days and extracted using RIPA assay. Protein quantified was measured using BCA assay. Briefly, proteins were used to analyze caspase-3 and caspase- 9 activity using Commercial ELISA kits (Nanjing Jiancheng Biology Engineering Institute).

Western blot analysis. Tissue extracts were prepared after treatment with gambogic acid at 28 days and extracted using RIPA assay. Protein quantified was measured using BCA assay. Briefly, proteins were size fractionated by $8-12 \%$ sodium dodecyl sulfate polyacrylamide gel electrophoresis and then transferred to a polyvinylidene difluoride membrane. The following antibodies were employed with matrix metalloproteinases (MMP)-2, MMP-9, NF-кB, phosphorylated (p-)p38, tissue inhibitors of matrix metalloproteases-1 (TIMP-1), PI3K, p-Akt and GAPDH (Santa Cruz Biotechnology Inc., Milan, Italy) at $4^{\circ} \mathrm{C}$ overnight. Secondary anti-mouse $\operatorname{IgG}$ peroxidase conjugate was used to incubate for $1 \mathrm{~h}$ at $37^{\circ} \mathrm{C}$. Detection was carried out with ECL Fast Pico (ECL-1002; Immunological Sciences, Rome, Italy) and quantified the ImageJ software system and Alliance LD (Uvitec, Cambridge, UK).

Statistical analysis. The data of statistical analysis are presented as mean \pm SD and were compared by two-way analysis of variance with a Sidak's multiple comparison test post hoc. P-value $<0.05$ considered to be statistically significant.

\section{Results}

Gambogic acid decreases arthritis scores in RA mice. To examine whether the effects of gambogic acid on arthritis, we

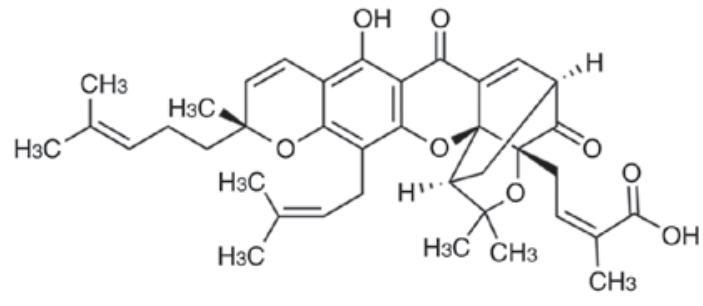

Figure 1. The structural formula of gambogic acid.

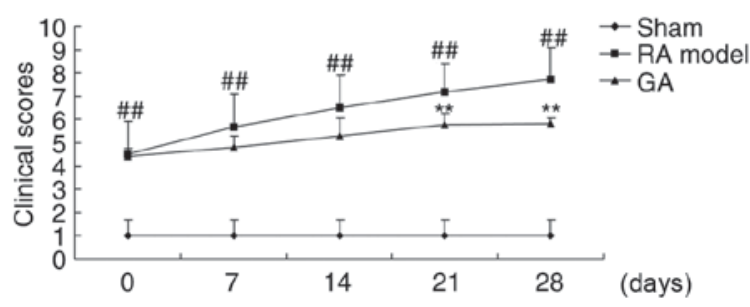

Figure 2. Gambogic acid decreased arthritis scores in RA mice. ${ }^{\# \#} \mathrm{P}<0.01$ compared with Sham, ${ }^{* *} \mathrm{P}<0.01$ compared with RA model. RA, rheumatoid arthritis; Sham, sham control group; RA model, RA model group; GA, gambogic acid group.

recorded arthritis scores in RA mice by gambogic acid. As showed in Fig. 1, a significant increase of arthritis scores in RA model mice. However, gambogic acid inhibited arthritis scores at 21 or 28 days in RA mice (Fig. 2).

Gambogic acid decreases TNF- $\alpha, I L-1 \beta, I L-6$ and $I L-18$ concentrations in RA mice. To investigate the anti-inflammation effects of gambogic acid on RA, TNF- $\alpha$, IL- $1 \beta$, IL- 6 and IL-18 concentrations were measured using ELISA KITS. Fig. 3 showed that TNF- $\alpha$, IL-1 $\beta$, IL- 6 and IL-18 concentrations in RA model mice were significantly increased, compared with control group. These inflammation factors were significantly reduced in RA mice by gambogic acid for 28 days (Fig. 3).

Gambogic acid decreases caspase-3 and caspase-9 activity in $R A$ mice. To assess the involvement of caspases for cell apoptosis of RA treated by gambogic acid, caspase- 3 and caspase- 9 activity were analyzed using ELISA kits. As showed that caspase- 3 and caspase- 9 activity of RA model was higher than those of control group (Fig. 4). The high level of caspase-3 and caspase-9 activity in RA model reduced by gambogic acid, compared with RA model group (Fig. 4).

Gambogic acid decreases MMP- 2 and MMP-9 protein expression in RA mice. Then, we analyzed MMP-2 and MMP-9 protein expression treated by gambogic acid. The result of Fig. 5 showed that RA induced MMP-2 and MMP-9 protein expression in mice model, compared with control group. Certainly, gambogic acid significantly inhibited MMP-2 and MMP-9 protein expression in RA model group, compared with RA model group (Fig. 5).

Gambogic acid decreases $N F-\kappa B$ and p-p38 protein expression in $R A$ mice. To understand the anti-inflammation mechanism of gambogic acid on RA, NF- $\mathrm{KB}$ and $\mathrm{p}-\mathrm{p} 38$ protein expression were measured using western blot analysis. As showed in 

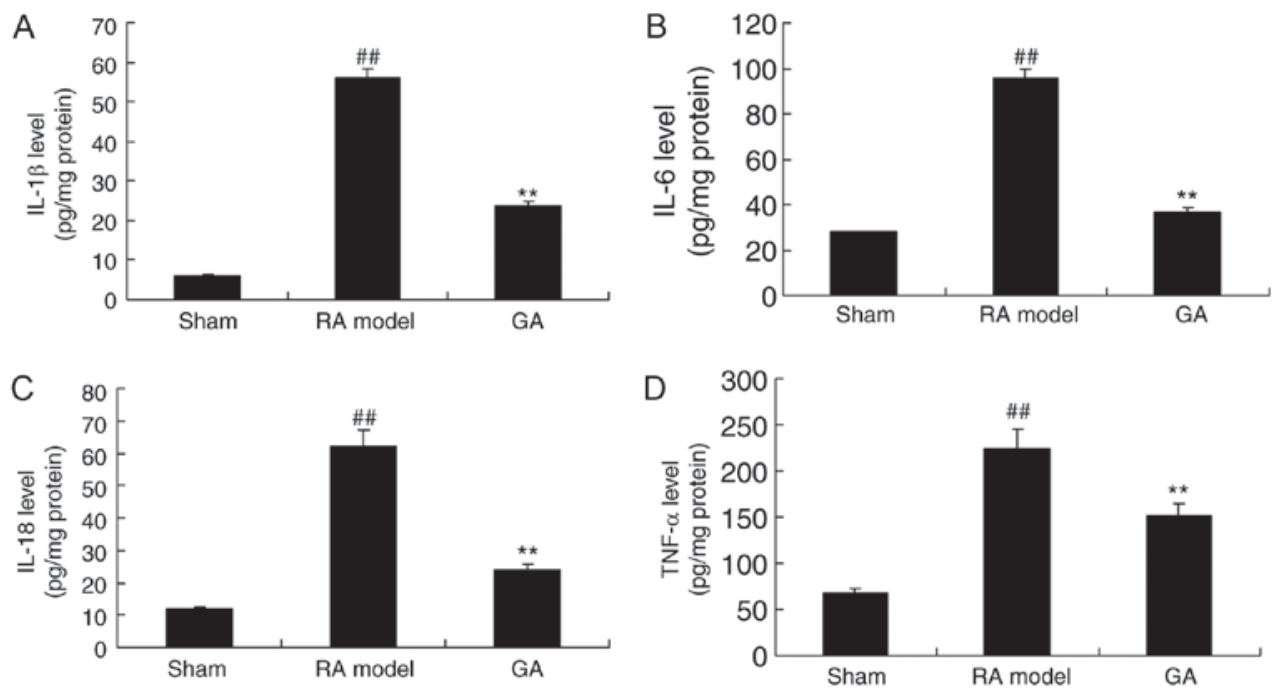

Figure 3. Gambogic acid decreased TNF- $\alpha$, IL-1 $\beta$, IL-6 and IL-18 concentrations in RA mice. Gambogic acid decreased (A) IL-1 $\beta$, (B) IL-6, (C) IL-18 and (D) TNF- $\alpha$, concentrations in RA mice. ${ }^{\# \#} \mathrm{P}<0.01$ compared with Sham, ${ }^{* *} \mathrm{P}<0.01$ compared with RA model. TNF, tumor necrosis factor; IL, interleukin; RA, rheumatoid arthritis; Sham, sham control group; RA model, RA model group; GA, gambogic acid group.
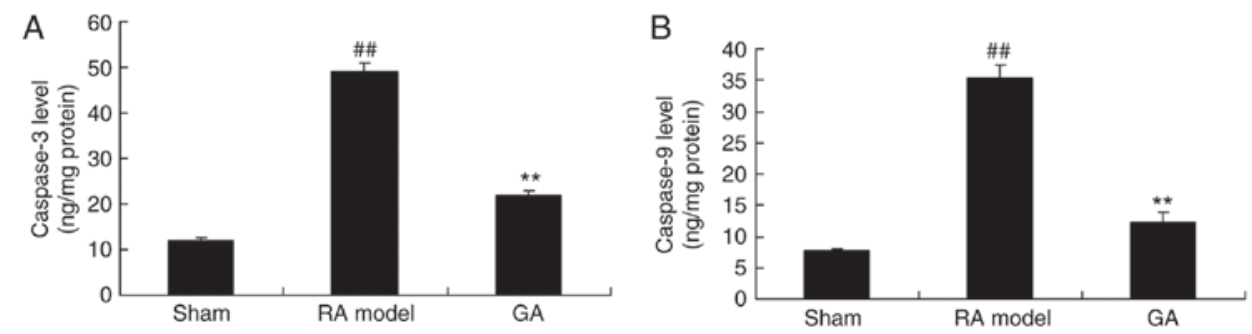

Figure 4. Gambogic acid decreased caspase-3 and caspase-9 activity in RA mice. Gambogic acid decreased (A) caspase-3 and (B) caspase-9 activity in RA mice. ${ }^{\# \#} \mathrm{P}<0.01$ compared with Sham, ${ }^{* *} \mathrm{P}<0.01$ compared with RA model. RA, rheumatoid arthritis; Sham, sham control group; RA model, RA model group; GA, gambogic acid group.
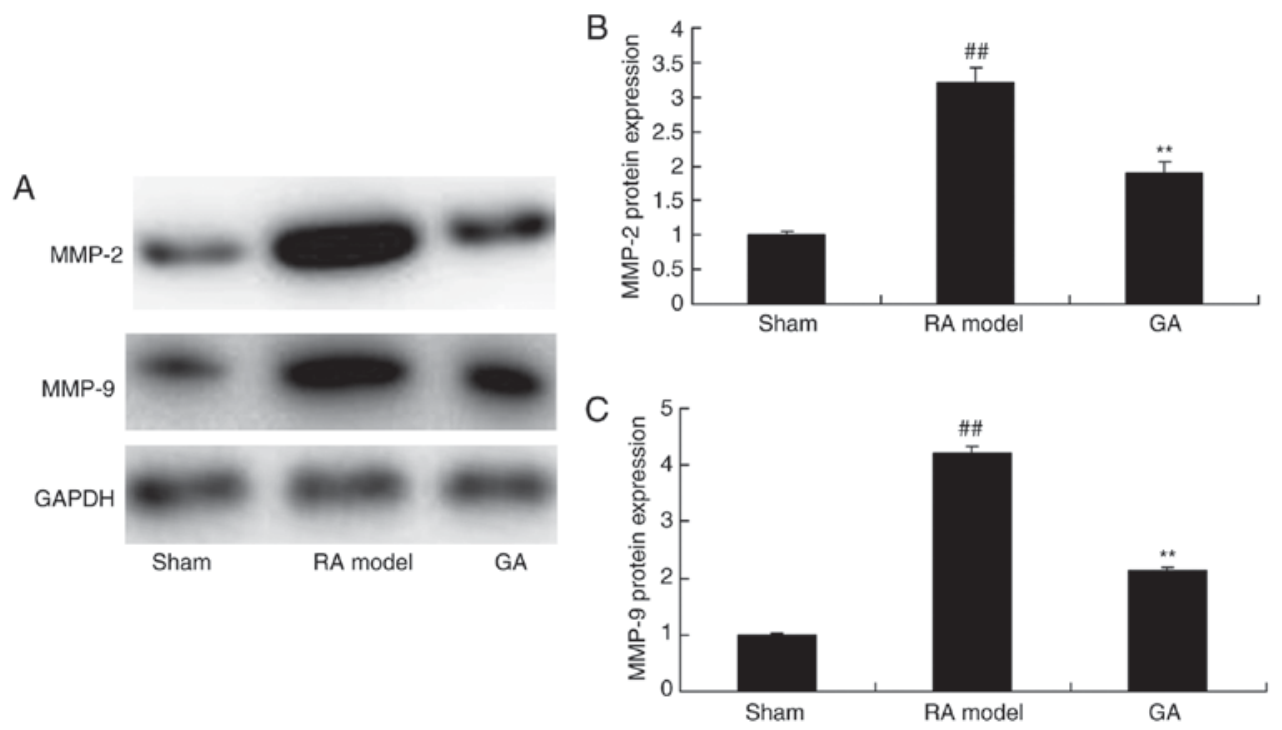

Figure 5. Gambogic acid decreased MMP-2 and MMP-9 protein expression in RA mice. Gambogic acid decreased MMP-2 and MMP-9 protein expression by (A) western blot assays and (B and C) statistical analysis of MMP-2 and MMP-9 protein expression in RA mice. ${ }^{\# \#} \mathrm{P}<0.01$ compared with Sham, ${ }^{* *} \mathrm{P}<0.01$ compared with RA model. MMP, matrix metalloproteinase; RA, rheumatoid arthritis; Sham, sham control group; RA model, RA model group; GA, gambogic acid group.

Fig. 6, NF- $\kappa \mathrm{B}$ and p-p38 protein expression were significantly induced in RA, model group, compared with control group.
Treatment with gambogic acid significantly suppressed NF- $\kappa \mathrm{B}$ and p-p38 protein expression in RA mice, exhibited that 

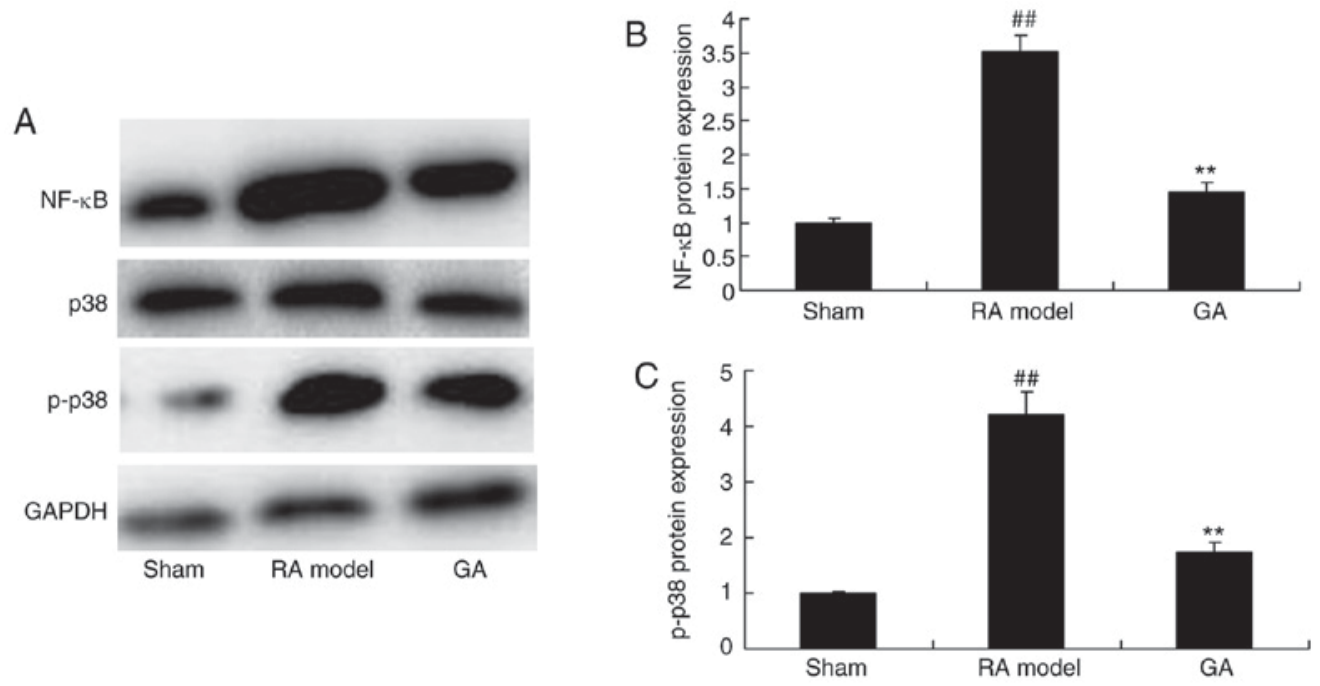

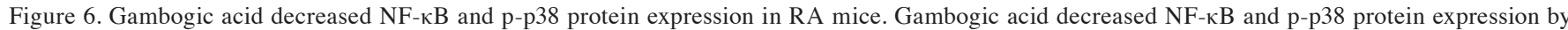
(A) western blot assays and (B and C) statistical analysis of NF- $\mathrm{kB}$ and p-p38 protein expression in RA mice. ${ }^{\# \#} \mathrm{P}<0.01$ compared with Sham, ${ }^{* *} \mathrm{P}<0.01$

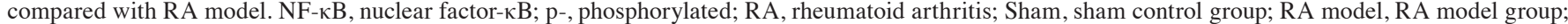
GA, gambogic acid group.
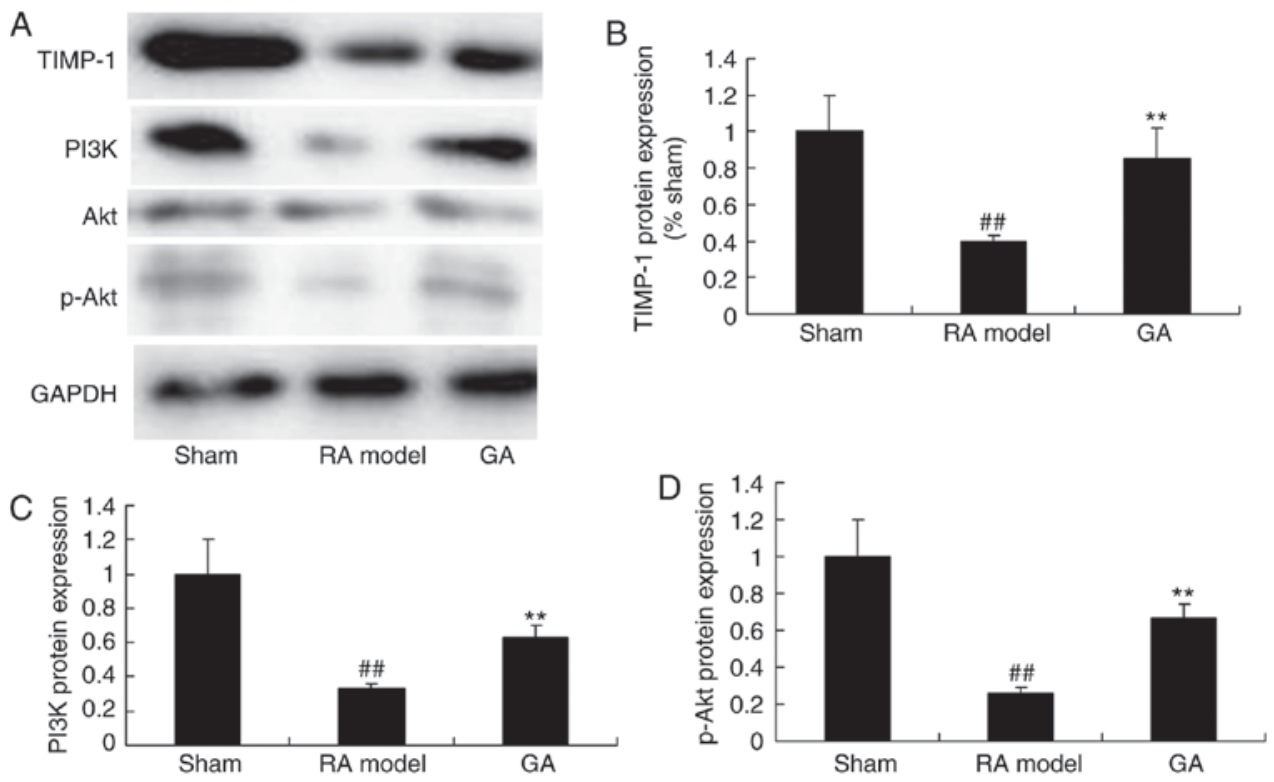

Figure 7. Gambogic acid increased TIMP-1, PI3K/Akt singnaling pathway in RA mice. Gambogic acid decreased TIMP-1, PI3K and p-Akt protein expression by (A) western blot assays and (B, C and D) statistical analysis of TIMP-1, PI3K and p-Akt protein expression in RA mice. ${ }^{\# \#} \mathrm{P}<0.01$ compared with Sham, ** $\mathrm{P}<0.01$ compared with RA model. TIMP-1, tissue inhibitor of matrix metalloprotease-1; PI3K, phosphoinositide 3-kinase; RA, rheumatoid arthritis; p-, phosphorylated; Sham, sham control group; RA model, RA model group; GA, gambogic acid group.

gambogic acid inhibited inflammation in RA mice through NF- $\kappa B$ and p-p38 protein expression (Fig. 6).

Gambogic acid increases PI3K/Akt singnaling pathway in RA mice. Furthermore, to investigate the anti-apoptosis mechanism of gambogic acid on RA, TIMP-1 and PI3K/Akt singnaling pathway were selected and analyzed in this study. Fig. 7 showed that TIMP-1 and PI3K/Akt singnaling pathway were suppressed in RA model group, compared with control group. Gambogic acid could promote TIMP-1 and PI3K/Akt singnaling pathway in RA mice. These data illuminated that gambogic acid alleviated cell apoptosis in RA through TIMP-1 and PI3K/Akt singnaling pathway.

\section{Discussion}

RA is a systemic inflammatory autoimmune disease related to the involvement of the surrounding joints, which is characterized by inflammation of the synovial tissue and progressive destruction of the joints (16). Cytokine TNF- $\alpha$ plays an important role in synovial inflammation and bone destruction of RA (17). It is suggested that TNF- $\alpha$ induces synovial cells to produce the expressions of many inflammatory cytokines (such as IL-6 and IL-8) and collagenase (MMP), which is a key factor for the persistence and progression of RA (18). In our study, gambogic acid inhibited arthritis scores, and TNF- $\alpha$, IL- $1 \beta$, IL-6 and IL-18 concentrations in RA mice. Wen et al (15) 
suggested that gambogic acid exhibits anti-psoriatic efficacy through inhibition of inflammation.

$\mathrm{NF}-\kappa \mathrm{B}$ signal pathway plays an important role in the inflammatory process of RA (19). The transcription factor, NF- $\kappa \mathrm{B}$ may play an important role in the pathogenesis of infectious diseases and autoimmune diseases by regulating the expression of various cytokines and cell adhesion molecules (6). The activated transcription factor, $\mathrm{NF}-\kappa \mathrm{B}$, binds to the $\kappa \mathrm{B}$ site in the promoter region of the corresponding inflammatory mediator target gene, leading to the over-expression of the inflammatory mediator gene (20). In recent years, we have found that NF- $\kappa \mathrm{B}$ is activated in RA synovial tissue abnormally, and the NF- $\kappa \mathrm{B}$ pathway in RA synovial tissue and synovial fluid regulates the expression of many inflammatory cytokines, such as cytokines (TNF- $\alpha$, IL-1, IL-6, IL-2, IL-12, IFN- $\gamma$ ), adhesion molecules (VCAM-1, ICAM-1), chemokines (IL-8, MIP-1 $\alpha$, MCP-1, TIMP-1), and MMPs (MMP-1, MMP-3, MMP-13) (21). And we also found that treatment with gambogic acid significantly suppressed NF- $\mathrm{B}$ and MMP-2 and MMP-9 protein expression in RA mice. Tang et al (11) suggested that gambogic acid inhibits the growth of ovarian cancer tumors through RELA/NF- $\mathrm{B}$ p65 (p65) activity. Qi et al (22) indicated that gambogic acid suppressed lung metastasis of human breast carcinoma cell through protein kinase $\mathrm{C}$ (PKC) mediated MMP-2/9 expression inhibition.

p38 mitogen-activated protein kinase plays an important role in normal cartilage cell physiology and pathogenesis of osteoarthritis (23). P38 can regulate the proliferation and survival of chondrocytes, balance the extracellular matrix metabolism, and play a key role in the pathogenesis of osteoarthritis cartilage degeneration induced by MMPs and proinflammatory factors (24). Our results indicated that gambogic acid significantly suppressed p-p38 protein expression in RA mice. Ma et al (13) demonstrated that gambogic acid inhibits osteoclast formation in p38 and Akt signalling pathways.

The abnormal mechanism of chondrocyte apoptosis has not been elucidated and may be related to many signal transduction pathways, in which PI3K/AKT signal transduction pathway has been considered as an important pathway of chondrocyte apoptosis (25). PI3K is a membrane protein that can directly or indirectly activate the downstream factor AKT by accepting the afferent signals of tyrosine kinase receptors, cytokine receptors, CD19, BCR, GPCR, etc. on the membrane (26). The biological effects of AKT include protein synthesis, anti-apoptosis, blocking the cytoplasm, regulating cell growth cycle, glucose metabolism (glycolysis, glucose conversion and glycogen synthesis) and neurodegeneration (27). The anti-apoptotic effect of AKT is realized by the following three ways: Playing anti-apoptotic effectby XIAP factor; inhibition of Bax, Bcl-2, Bim and FOX01-induced apoptosis; isolation of $\mathrm{Bad}$ (14-3-3) in the cytoplasm (27). $\mathrm{PI} 3 \mathrm{~K} /$ Akt signal is involved in the regulation of B lymphocyte proliferation and differentiation by the activation of mTOR, and mTOR is a target molecule for rapamycin, which plays a critical role in regulating cell growth and proliferation (28). mTOR signal affects cell cycle, cell growth and cell proliferation. In many human cancers, the absence of some important tumor suppressors (PTEN, TSC1/2, LKB1) in mTOR signal pathway, cell mutations or the gene amplification in PI3CA
(P110 $\alpha$ subtype) and abnormalities caused by Akt activation mutations, ultimately lead to cell proliferation, cell survival and cell self-phagocytosis inhibition $(8,29)$. Our data also showed that gambogic acid could promote PI3K/Akt singnaling pathway in RA mice. Wang et al (30) identified that gambogic acid suppresses multiple myeloma cell growth through PI3K/Akt singnaling pathway.

In summary, this study reveals that gambogic acid prevents inflammation and apoptosis in RA model mice through $\mathrm{NF}-\kappa \mathrm{B}$ p65/MMP-2/9 and PI3K/Akt singnaling pathway. These findings may be valuable in the further exploration of gambogic acid in therapy for RA.

\section{References}

1. Bijlsma JW, Welsing PM, Woodworth TG, Middelink LM, Pethö-Schramm A, Bernasconi C, Borm ME, Wortel CH, ter Borg EJ, Jahangier ZN, et al: Early rheumatoid arthritis treated with tocilizumab, methotrexate, or their combination (U-Act-Early): A multicentre, randomised, double-blind, double-dummy, strategy trial. Lancet 388: 343-355, 2016.

2. Sellam J, Marion-Thore S, Dumont F, Jacques S, Garchon HJ, Rouanet S, Taoufik Y, Hendel-Chavez H, Sibilia J, Tebib J, et al: Use of whole-blood transcriptomic profiling to highlight several pathophysiologic pathways associated with response to rituximab in patients with rheumatoid arthritis: Data from a randomized, controlled, open-label trial. Arthritis Rheumatol 66: 2015-2025, 2014.

3. Tanaka Y, Kawai S, Takeuchi T, Yamamoto K and Miyasaka N: Prevention of joint destruction by tacrolimus in patients with early rheumatoid arthritis: A post hoc analysis of a double-blind, randomized, placebo-controlled study. Mod Rheumatol 23: 1045-1052, 2013.

4. Li R, Zhao JX, Su Y, He J, Chen LN, Gu F, Zhao C, Deng XR, Zhou W, Hao YJ, et al: High remission and low relapse with prolonged intensive DMARD therapy in rheumatoid arthritis (PRINT): A multicenter randomized clinical trial. Medicine (Baltimore) 95: e3968, 2016.

5. Jia Q, Cheng W, Yue Y, Hu Y, Zhang J, Pan X, Xu Z and Zhang P: Cucurbitacin E inhibits TNF- $\alpha$-induced inflammatory cytokine production in human synoviocyte MH7A cells via suppression of PI3K/Akt/NF-kB pathways. Int Immunopharmacol 29: 884-890, 2015.

6. Cho ML, Ju JH, Kim KW, Moon YM, Lee SY, Min SY, Cho YG, Kim HS, Park KS, Yoon $\mathrm{CH}$, et al: Cyclosporine A inhibits IL-15-induced IL-17 production in $\mathrm{CD}^{+} \mathrm{T}$ cells via down-regulation of PI3K/Akt and NF-kappaB. Immunol Lett 108: 88-96, 2007.

7. Lv Q, Zhu XY, Xia YF, Dai Y and Wei ZF: Tetrandrine inhibits migration and invasion of rheumatoid arthritis fibroblast-like synoviocytes through down-regulating the expressions of Rac1, Cdc42, and RhoA GTPases and activation of the PI3K/Akt and JNK signaling pathways. Chin J Nat Med 13: 831-841, 2015.

8. Kim TH, Choi SJ, Lee YH, Song GG and Ji JD: Combined therapeutic application of mTOR inhibitor and vitamin D(3) for inflammatory bone destruction of rheumatoid arthritis. Med Hypotheses 79: 757-760, 2012.

9. Zhang L, Yi Y, Chen J, Sun Y, Guo Q, Zheng Z and Song S: Gambogic acid inhibits Hsp90 and deregulates TNF- $\alpha / \mathrm{NF}-\mathrm{kB}$ in HeLa cells. Biochem Biophys Res Commun 403: 282-287, 2010.

10. Palempalli UD, Gandhi U, Kalantari P, Vunta H, Arner RJ, Narayan V, Ravindran A and Prabhu KS: Gambogic acid covalently modifies IkappaB kinase-beta subunit to mediate suppression of lipopolysaccharide-induced activation of NF-kappaB in macrophages. Biochem J 419: 401-409, 2009.

11. Tang Q, Lu M, Zhou H, Chen D and Liu L: Gambogic acid inhibits the growth of ovarian cancer tumors by regulating p65 activity. Oncol Lett 13: 384-388, 2017.

12. Geng J, Xiao S, Zheng Z, Song S and Zhang L: Gambogic acid protects from endotoxin shock by suppressing pro-inflammatory factors in vivo and in vitro. Inflamm Res 62: 165-172, 2013.

13. Ma J, Ma Y, Liu X, Chen S, Liu C, Qin A and Fan S: Gambogic acid inhibits osteoclast formation and ovariectomy-induced osteoporosis by suppressing the JNK, p38 and Akt signalling pathways. Biochem J 469: 399-408, 2015. 
14. Ito H, Noda K, Yoshida K, Otani K, Yoshiga M, Oto Y, Saito S and Kurosaka D: Prokineticin 2 antagonist, PKRA7 suppresses arthritis in mice with collagen-induced arthritis. BMC Musculoskelet Disord 17: 387, 2016.

15. Wen J, Pei H, Wang X, Xie C, Li S, Huang L, Qiu N, Wang W, Cheng $X$ and Chen L: Gambogic acid exhibits anti-psoriatic efficacy through inhibition of angiogenesis and inflammation. J Dermatol Sci 74: 242-250, 2014

16. Lal P, Su Z, Holweg CT, Silverman GJ, Schwartzman S, Kelman A, Read S, Spaniolo G, Monroe JG, Behrens TW and Townsend MJ: Inflammation and autoantibody markers identify rheumatoid arthritis patients with enhanced clinical benefit following rituximab treatment. Arthritis Rheum 63: 3681-3691, 2011.

17. Nair SC, Welsing PM, Jacobs JW, van Laar JM, Rensen WH, Ardine de Wit G, Bijlsma JW and Lafeber FP: Economic evaluation of a tight-control treatment strategy using an imaging device (handscan) for monitoring joint inflammation in early rheumatoid arthritis. Clin Exp Rheumatol 33: 831-838, 2015.

18. Kullich WC, Mur E, Aglas F, Niksic F and Czerwenka C: Inhibitory effects of leflunomide therapy on the activity of matrixmetalloproteinase-9 and the release of cartilage oligomeric matrix protein in patients with rheumatoid arthritis. Clin Exp Rheumatol 24: 155-160, 2006.

19. Min HK, Kim SM, Baek SY, Woo JW, Park JS, Cho ML, Lee J, Kwok SK, Kim SW and Park SH: Anthocyanin extracted from black soybean seed coats prevents autoimmune arthritis by suppressing the development of Th17 cells and synthesis of proinflammatory cytokines by such cells, via inhibition of NF-кB. PLoS One 10: e0138201, 2015.

20. Liu XZ, Fan J, Qi K, Liu SP, Xu WD, Gao Y, Gu XD, Li J, Bai CG, Shi YQ, et al: Dishevelled2 promotes apoptosis and inhibits inflammatory cytokine secretion in rheumatoid arthritis fibroblast-like synoviocytes through crosstalk with the NF- $\kappa \mathrm{B}$ pathway. Oncotarget 8: 12649-12663, 2017.

21. Li Y, Wang LM, Xu JZ, Tian K, Gu CX and Li ZF: Gastrodia elata attenuates inflammatory response by inhibiting the NF- $\kappa B$ pathway in rheumatoid arthritis fibroblast-like synoviocytes. Biomed Pharmacother 85: 177-181, 2017.

22. Qi Q, Gu H, Yang Y, Lu N, Zhao J, Liu W, Ling H, You QD Wang $\mathrm{X}$ and Guo Q: Involvement of matrix metalloproteinase 2 and 9 in gambogic acid induced suppression of MDA-MB-435 human breast carcinoma cell lung metastasis. J Mol Med (Berl) 86: 1367-1377, 2008
23. Li CH, Xu LL, Zhao JX, Sun L, Yao ZQ, Deng XL, Liu R, Yang L, Xing R and Liu XY: CXCL16 upregulates RANKL expression in rheumatoid arthritis synovial fibroblasts through the JAK2/STAT3 and p38/MAPK signaling pathway. Inflamm Res 65: 193-202, 2016.

24. Kim HR, Park MK, Cho ML, Kim KW, Oh HJ, Park JS, Heo YM, Lee SH, Kim HY and Park SH: Induction of macrophage migration inhibitory factor in ConA-stimulated rheumatoid arthritis synovial fibroblasts through the P38 map kinase-dependent signaling pathway. Korean J Intern Med 25: 317-326, 2010.

25. Han W, Xiong Y, Li Y, Fang W, Ma Y, Liu L, Li F and Zhu X: Anti-arthritic effects of clematichinenoside (AR-6) on PI3K/Akt signaling pathway and TNF- $\alpha$ associated with collagen-induced arthritis. Pharm Biol 51: 13-22, 2013.

26. Liu Y, Pan YF, Xue YQ, Fang LK, Guo XH, Guo X, Liu M, Mo BY, Yang MR, Liu F, et al: uPAR promotes tumor-like biologic behaviors of fibroblast-like synoviocytes through PI3K/Akt signaling pathway in patients with rheumatoid arthritis. Cell Mol Immunol, Jan 16, 2017 (Epub ahead of print).

27. Yuan FL, Xu RS, Jiang DL, He XL, Su Q, Jin C and Li X: Leonurine hydrochloride inhibits osteoclastogenesis and prevents osteoporosis associated with estrogen deficiency by inhibiting the NF- $\mathrm{KB}$ and PI3K/Akt signaling pathways. Bone 75: 128-137, 2015.

28. Malemud CJ: The PI3K/Akt/PTEN/mTOR pathway: A fruitful target for inducing cell death in rheumatoid arthritis? Future Med Chem 7: 1137-1147, 2015.

29. Mitra A, Raychaudhuri SK and Raychaudhuri SP: IL-22 induced cell proliferation is regulated by $\mathrm{PI} 3 \mathrm{~K} / \mathrm{Akt} / \mathrm{mTOR}$ signaling cascade. Cytokine 60: 38-42, 2012.

30. Wang F, Zhang W, Guo L, Bao W, Jin N, Liu R, Liu P, Wang Y, Guo Q and Chen B: Gambogic acid suppresses hypoxiainduced hypoxia-inducible factor- $1 \alpha /$ vascular endothelial growth factor expression via inhibiting phosphatidylinositol 3-kinase/ $\mathrm{Akt} / \mathrm{mammalian}$ target protein of rapamycin pathway in multiple myeloma cells. Cancer Sci 105: 1063-1070, 2014. 